\title{
Research on Oxygen Concentration in the Clean Room of Semiconductor Factory Based on FLUENT
}

\author{
Bo Meng \\ College of Land and Environment, Shenyang Agricultural University, Shenyang 110866, China
}

\begin{abstract}
This paper simulates the diffusion of nitrogen in clean room. In GAMBIT, we establish a three-dimensional model, then set parameters in FLUENT. Finally, the FLUENT post-processing function and TECPLOT obtain the velocity vector diagram, nitrogen andoxygen distribution diagram and anoxic zone distribution map. The research shows that changing the ventilation structure can reduce the range of the hypoxia area thus ensure the health and safety of the work.
\end{abstract}

Keywords: FLUENT, semiconductor, clean room, oxygen concentration

\section{Introduction}

With the continuous development of the semiconductor industry, we also need to see the potential hazards. Due to the requirements of the production process, the integrated circuit industry needs to use more than 300 kinds of raw materials and solvents. Corrosive, toxic and flammable chemicals and a variety of hazardous equipment are used in the manufacturing process. These chemicals and industrial instruments pose a threat to the human body, easily lead to occupational disease. The appearance of occupational disease symptoms occurs after a period of dose accumulation. According to the "Occupation Hazards Catalog" issued by China's Ministry of Health, in semiconductor manufacturing there are at least the causes of occupational diseases as follows: chemical factors, radioactive substances, physical factors and risk factors that causes skin disease [1].

Meanwhile, the semiconductor
manufacturing
environmental pollution. It needs to
electroplate a layer of tin or tin-lead alloy in order that copper pins are well welded to the PCB board. Therefore, the electroplating wastewater contains heavy metals in semiconductor industry. Noise and hazardous wastes will also pollute the environment [2].

In addition to the above two aspects of the harm, because the chips should be produced in the sealed clean room, once on fire, it is likely to happen evacuation route blockage and poor ventilation, resulting in a large number of casualties. There are also the dangers of mechanical damage, confined space operations and high altitude crashes, etc. Therefore, there are many risk factors in the semiconductor industry.

\section{Models and Methods}

\subsection{Computational fluid dynamics model}

Computational fluid dynamics (CFD) is a new independent discipline calculation method based on the classical fluid dynamics and numerical simulation. The basic principle is to use computer to run numerical calculation and image display, and then study the physical phenomena such as fluid flow and heat transfer. It has made many breakthroughs theoretically and practically, and has solved many 
complicated problems of flow and heat transfer in modern science[1,6]. The basic principle of computational fluid mechanics on analysis of the flow and heat transfer, is composed of a finite number of variables of discrete points, such as continuous quantities of concentration field and velocity field in spatial domain, and then construct the equations of variable relationship on discrete points, and finally get the spatial distribution of the basic physical flow quantity, and how it changes over time[7]. The development of CFD simulation is closely related to the development of computer technology. As early as 1970 s, the CFD software was given birth. However due to the limitation of computer memory and computing power, it has not been widely used until last decade.

\subsection{FLUENT simulation platform}

FLUENT is one of the most advanced commercial CFD software, which is widely used in fluid calculation. American company FLUENT Inc. released it in 1983. FLUENT is used to simulate and study the fluid flow and heat transfer phenomena in complex geometry. It supports a variety of grid formats, users can choose to use structured and unstructured grid according to their own needs. For example, in a two-dimensional problem, the user can select a triangular mesh or a quadrilateral mesh. In a three-dimensional problem, users can choose a tetrahedron, hexahedron, pyramid, wedge and polyhedral mesh; the hybrid grid can also be selected[8-10]. This study applies CFD as the core theory, with the help of FLUENT simulation platform to complete the simulation of the airflow diffusion[11, 12].

3 Methodology

\subsection{Study area}

In this paper, the clean room is the semiconductor packaging workshop. The whole clean room measures $55.2 \mathrm{~m}$ of width,
$64.8 \mathrm{~m}$ of length, and $3.048 \mathrm{~m}$ of height, including three kinds of nitrogen release sources: the processing machines, nitrogen cabinets, and feed inlets. Except for the northwest of clean room, there are a large number of nitrogen sources in other places.In the simulation of clean room, nitrogen devices are widely used, the release rate and the release areas of different devices are not the same, and the quantity of different devices are differentiated as well. The amount of devices is at least 1 , up to 39 . The maximum release area is 4000 times as large as the smallest release port. The maximum release rate is 8888 times as speedy as the smallest. These differences add difficulties to the simulation. In the table the first 13 kinds of devices are a variety of reaction machines, when the machines are running, there will be the release of nitrogen. In the nitrogen cabinets and feed inlets, nitrogen is as protective gas. Nitrogen is rarely released when the door is closed; while it will release a large number of nitrogen when open the door.

Clean room fresh air is pressed into from air inlet on the above, and the wind is flowed out through air outlet by natural ventilation. The outflow of the gas from the clean room is mixed with the fresh air according to the proportion of $90 \%$ and $10 \%$, and then is fed into the clean room by the air inlet to realize the gas circulation of the whole clean room. The air inlets and the air outlets are both in the ceiling, which reduces the bottom air circulation, and is not conducive to the diffusion of nitrogen into the air. After the clean room engineer determined, in air inlet the wind velocity is $0.3 \mathrm{~m} / \mathrm{s}$. The inlet air temperature in summer and winter is slightly different, we take the average temperature as $16^{\circ} \mathrm{C}$. The simulation of the clean room is the front of a semiconductor factory; the mapping is shown in Fig.1. 


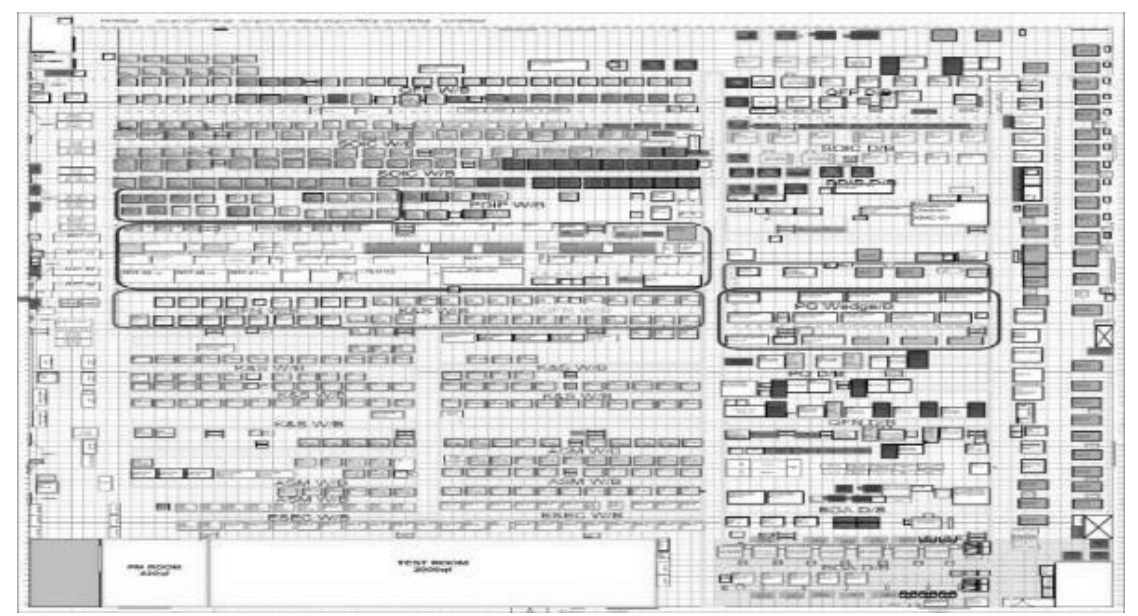

Fig. 1. Simulation of clean room layout

The boxes filled with color in Fig 1 indicate devices using nitrogen. Different colors represent different equipment. A colorless box indicates a device that does not use nitrogen. There are 11 feeding ports on the left side of the clean room, which are the entrances of the intermediate products produced in the production process in two rooms. Nitrogen is used as a protective gas inside. The gray boxes represent the floor, each of which is a square of side length $0.6 \mathrm{~m}$. The distance between the devices can be determined by the relative positions of the equipment and the floor.

\subsection{Boundary conditions and simulation parameter setting}

The boundary conditions and simulation parameters setting follows the steps in below:

(1) Grid-related operation

Enter mesh file in FLUENT. First of all, run the Check command to check the grid, if there is a negative volume in the grid, re-dividing the grid in GAMBIT is needed.

(2) Select the model

Because simulation of nitrogen leakage distribution is a balanced state in the clean room, it only needs to focus on the distribution of different gas concentration. Choose the "Steady" simulation; the others can be in accordance with the default settings.
(3)Modify material properties

Because the simulation is to study the nitrogen and oxygen concentration distribution in the clean room, and the air also contains these two kinds of gas, it needs to consider the air as mixed gas of nitrogen, oxygen and carbon dioxide, and to ignore other rare gas components.

(4) Set boundary conditions

The simulation of all the nitrogen machines sets to run at the same time, the leakage of nitrogen begins at the same time. This situation is the most dangerous, known as extreme leakage state.

(5) Calculation initialization and settings for other controlling parameters

Since the density of nitrogen is less than that of air, gravity should be considered in simulation.

Then start the iterative calculation. When the residuals are no longer declined in the residual graph, the operation has been proved to be convergent. Stop the calculation. The calculation results can be obtained and then analyze the results.

\section{Simulation Results}

\subsection{Movement analysis of airflow}

Firstly, the velocity vector diagram of XY 
plane in the $\mathrm{Z}$-axis section of the whole clean room was observed. Select $z=1.6 \mathrm{~m}$, that is, the rate vector of human body respiration height, as shown in Fig. 2.

It can be seen from Figure 3 that the maximum flow rate is from some of nitrogen device sources, the general rate reaches more than $0.3 \mathrm{~m} / \mathrm{s}$, and some can reach more than $0.5 \mathrm{~m} / \mathrm{s}$. The large speed areas are under the air inlets and outlets. Under the inlets, the velocity is generally $0.2 \mathrm{~m} / \mathrm{s}$ to $0.3 \mathrm{~m} / \mathrm{s}$, while under the outlets, the velocity reaches $0.1 \mathrm{~m} / \mathrm{s}$ to $0.2 \mathrm{~m} / \mathrm{s}$. Most of the other regions are below $0.01 \mathrm{~m} / \mathrm{s}$. In the upper left corner and the lower right corner of the clean room, because one side is blocked by the wall of the clean room, while the other sides of the two areas are the machine, two major vortexes are formed. Therefore, in the horizontal direction, although the airflow rate is very low, but the direction is complex, at the same time, the scale of movement of air is not large; it is in a very small range. Take the $y=-23.4 \mathrm{~m}$ plane, the plane in the three-dimensional model of the position is shown in Figure 1. The plane is just in front of three nitrogen release sources, so the plane can be very suitable to observe the movement of nitrogen from the release port into the clean

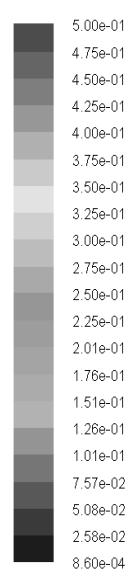

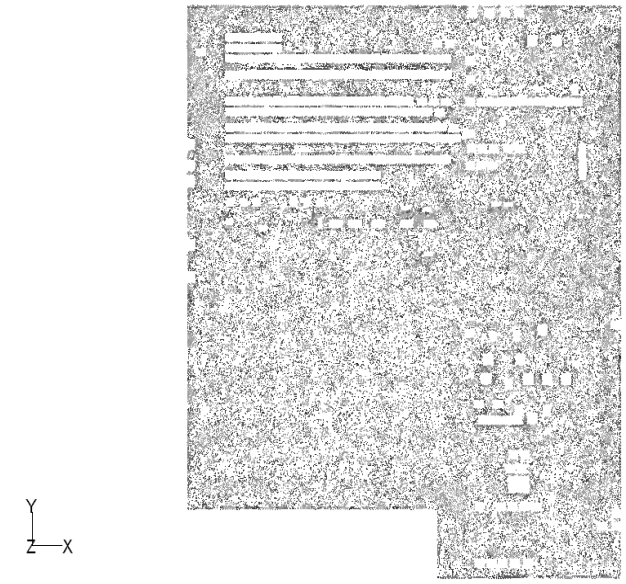

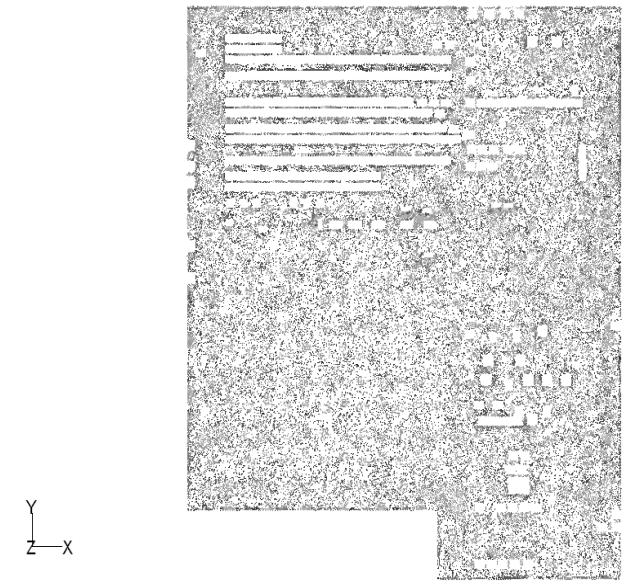

Fig. 2. $z=1.6 \mathrm{~m}$ plane velocity vector diagram

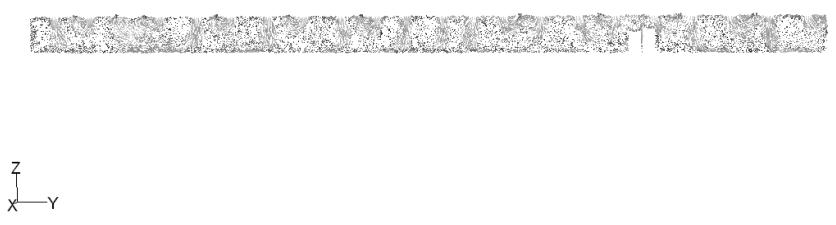

Fig.3. $\mathrm{x}=-21 \mathrm{~m}$ plane velocity vector diagram 
room in the vertical direction. At the same time, the plane is close to the air inlets, so the dilution effects of fresh air entering through the air inlets can also be observed.

As shown in Fig3, the inlet velocity in the FLUENT boundary conditions is set to $0.3 \mathrm{~m} / \mathrm{s}$, and the outlet velocity is higher than the air inlet, some places can reach more than $0.8 \mathrm{~m} / \mathrm{s}$. When the inlet and outlet are close, the majority of air getting into the clean room from the inlet goes out of the clean room by the upper part of the clean room from outlet, so few nitrogen dilution effects occur in the clean room. As can be seen in fig 5 and fig6, vertical air flow movement is very active in the clean room while the airflow directions are disordered, which is not conducive for dust particles to leave the clean room. Due to the saving cost strategy of clean room, air inlets and outlets are all designed on the ceiling of clean room, clean ventilation efficiency is immensely reduced.

\subsection{Analysis of gas concentration distribution}

TECPLOT software provides a 3D nitrogen and oxygen concentration distribution figure of the clean room. The simulation in Fig4 shows that the low oxygen concentration areas are the same with the areas of high nitrogen concentration, which is mainly concentrated in the central and southeastern of clean room. In extreme conditions, there are a lot of areas with low oxygen concentration, especially in the eastern part of clean room.

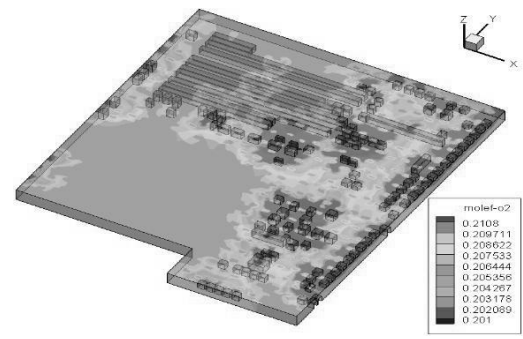

Fig. 4.Three-dimensional distribution of oxygen volume fraction in extreme state
In FLUENT simulation, due to the conversion of mass fraction into volume fraction, the oxygen content in the air inlet on the ceiling of the clean room is set to $21.08 \%$, slightly higher than the atmospheric oxygen content of $20.9 \%$. At the same time, the gas in the air inlet is composed of $90 \%$ circulating gas and $10 \%$ fresh air, the oxygen content is lower than that of the fresh air. Taking into account these two points, the simulation results in the gas should be slightly higher than the actual situation; the error is in the $0.2 \%$ range. So for safety reasons, the hypoxia standard should be increased from $19.5 \%$ to $19.7 \%$. The hypoxia distribution in each direction section of the clean room in FLUENT is shown below:

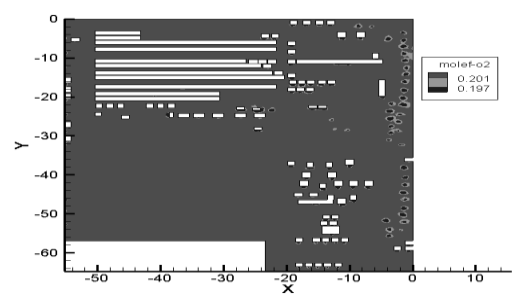

Fig. 5. Hypoxia area diagram of $\mathrm{z}=1.6$ plane

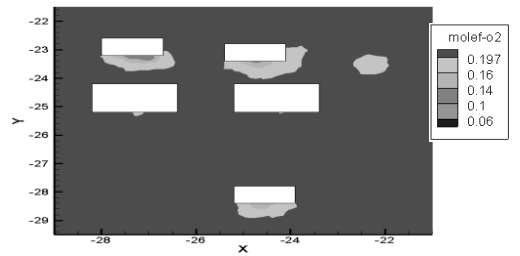

Fig. 6. Hypoxia range distribution map with wind speed of $0.3 \mathrm{~m} / \mathrm{s}$ (local)

As can be seen from the Fig5-6, most of the oxygen content in the clean room achieves the standards, meanwhile besides the central region, the hypoxia is concentrated in the vicinity of nitrogen release devices. After the measurement, the range of hypoxia is from the release port to the release direction $0.77 \mathrm{M}$. Combined with the velocity vector analysis we can draw the conclusion that, most nitrogen release is on horizontal direction initially, then to vertical motion. 


\subsection{Change the ventilation structure of the clean room}

Based on the simulation results, we discuss the method of increasing the production safety by changing the ventilation structure of the clean room, and the corresponding simulation results are obtained by FLUENT simulation.

The simulation results show that under extreme conditions, clean room hypoxic areas are from the leak port at $0.77 \mathrm{~m}$ maximum, while the operator standing position generally is less than $0.5 \mathrm{~m}$ from the machine. With the development of enterprises, it is necessary to strengthen the production capacity of clean room. So in order to ensure the clean room air quality, it is inevitable to increase the ventilation of the clean room. Clean room ventilation system includes turbulent flow, laminar flow, and composite flow. Laminar flow leads to regular air flow, and good cleaning effect. The clean room ceiling in the study applies turbulent flow ventilation with grille inlet and outlet of the wind, which affects the dilution of nitrogen from the machine, and influences the air quality in the clean room.

This study reduces the indoor hypoxia area by changing the distribution of clean room air outlet to change the airflow form. Clean room ventilation structure will be changed as air inlet on the ceiling and air outlet by the floor. Compared to the original ventilation, clean airflow disturbance degree is widely decreased. Under the extreme condition of the simulation, keeping the other sets unchanged, the original "outlet" is changed to "wall" type, and the "ground" will be changed to "pressure-out" type. Through iterative calculation by FLUENT, hypoxic areas at the respiratory plane after changing the clean room ventilation structure is shown in Fig7.

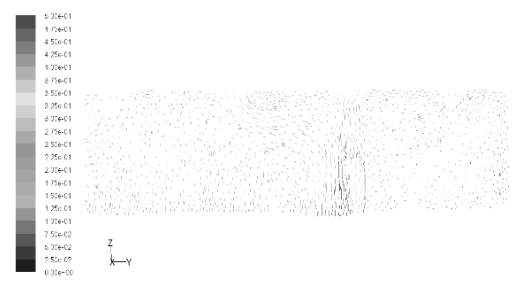

Fig.7. After changing clean room structure $x=-21 \mathrm{~m}$ velocity vector

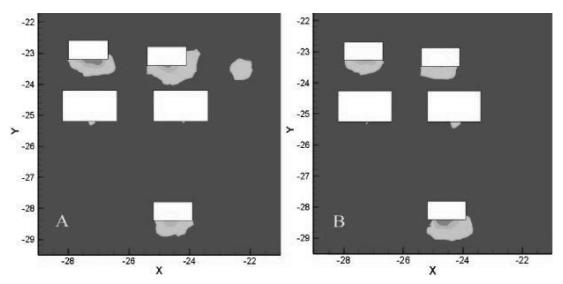

Fig.8. Comparison of the hypoxia area distribution of $\mathrm{z}=1.6 \mathrm{~m}$ profiles before and after changing the clean room structure

As can be seen from Fig7-8, the area of hypoxia on the respiratory plane of the clean room is greatly reduced when the structure of the clean room is changed (air inlet on the top of the clean room, and air outlet on the floor). Especially, on the RSW upper half circular, hypoxia zone crucially disappeared, while, there are still some hypoxic areas in the vicinity of the leak port of nitrogen machine.

\section{Conclusion}

(1) This paper simulates the diffusion of nitrogen in the clean room, based on the FLUENT of CFD software, in order to analyze the size of hypoxia in the clean room. Through the research, the application of FLUENT software can be a good simulation of the diffusion of nitrogen and hypoxia range, which provides support for the clean room safety management, and protects the health of the clean room workers.

(2) This paper studies the oxygen distribution in the clean room. The maximum distance from the anoxic zone to the releasing port was $0.77 \mathrm{M}$. In the vertical direction, the hazard ratio is greater than in the horizontal 
direction. In practical applications, do not operate the devices on the top of the machine as far as possible. At the same time, according to the simulation results, it can be divided into different levels of risk distances. It stipulates different exposure time for different risk distances, in order to ensure the health and safety of workers.

(3) The paper studies the effectiveness of the method to reduce the hypoxia range by changing the structure of clean room. It simulates the change of the hypoxia areas in the clean room under the condition of $0.3 \mathrm{~m} / \mathrm{s}$, $0.4 \mathrm{~m} / \mathrm{s}, 0.5 \mathrm{~m} / \mathrm{s}, 0.6 \mathrm{~m} / \mathrm{s}$ and $0.7 \mathrm{~m} / \mathrm{s}$ when the air inlets are on the top of the clean room. The simulation result shows that the change of clean room structure, the outlet from the ceiling to the ground, can significantly reduce the clean room hypoxia area, the maximum leakage distance from hypoxic area turns to $0.48 \mathrm{~m}$.

\section{Acknowledgements}

This paper was supported by The Ministry of education, Humanities and Social Science Foundation of China(No:13YJCZH129) and The PhD Start-up Fund of Natural Science Foundation of Liaoning Province, China (No: 20131099). The authors deeply appreciate the support.

\section{References}

[1] $\mathrm{Li} \mathrm{J}$, et al. The unfolding of '12.23' Kaixian blowout accident in China. Safety Science, 2009, 47(8): 1107-1117.

[2] Li J, et al., The application of FLUENT in simulating outcomes from chlorine leakage accidents in a typical chemical factory. Toxicology And Industrial Health, 2016, 32(5): 919-935.

[3] Xu T. Characterization of minienvironments in a clean room: Design characteristics and environmental performance. Building and Environment, 2007, 42(8): 2993-3000.
[4] Xu T. Characterization of minienvironments in a cleanroom: Assessing energy performance and its implications. Building and Environment, 2008, 43(9): 1545-1552.

[5] Chen S, et al., Dispersion of gas pollutant in a fan-filter-unit (FFU) cleanroom. Building and Environment, 2007, 42(5): 1902-1912.

[6] Li J, et al. Numerical simulation of the large-scale malignant environmental pollution incident. Process Safety and Environmental Protection, 2009, 87(4): 232-244.

[7] Hassan A A, Crowther J M. Modelling of fluid flow and pollutant dispersion in a street canyon. Environmental Monitoring and Assessment, 1998, 52(1-2): 281-297.

[8] Shih H, et al., Simulation and testing of pollutant dispersion during preventive maintenance in a cleanroom. Building and Environment, 2009, 44(12): 23192326.

[9] Noh K, Kim H, Oh M. Study on contamination control in a minienvironment inside clean room for yield enhancement based on particle concentration measurement and airflow CFD simulation. Building and Environment, 2010, 45(4):825-831.

[10] Hu SC, Chuah Y K, Yen M C. Design and evaluation of a minienvironment for semiconductor manufacture processes. Building and Environment, 2002, 37(2): 201-208.

[11] Sadjadi R, Liu B. Supply plenum and air-flow uniformity in clean rooms. Journal of the IES, 1991, 34(2): 56-60.

[12] Chen J, et al., The development of fan filter unit with flow rate feedback control in a cleanroom. Building and Environment, 2007, 42(10): 3556-3561. 